\title{
Role of autophagy in the progress of coronary total occlusion
}

\author{
Demircan $\mathrm{G}^{1}$, Kaplan $\mathrm{O}^{2}$, Ozdas $\mathrm{SB}^{1}$ \\ Department of Medical Biology and Genetics Istanbul Bilim University, Istanbul, Turkey. \\ drozgurkaplan@yahoo.com
}

\section{ABSTRACT}

OBJECTIVE: In this study, we measured the level of autophagy enzyme in patients with coronary artery disease (CAD) and investigated whether the role of autophagy existed in the progress of coronary collateral and coronary total occlusion (TO).

METHODS: Overall, 115 participants were included in this study. They were divided into the three groups: group 1: patients had chronic TO $(n=49)$; group 2: patients had acute TO such as myocardial infarction $(n=36)$; and group 3: participants were normal controls $(n=30)$. The enzyme-linked immunosorbent assay (ELISA) kit for autophagy-related protein 5 (ATG5) in the plasma was studied for these three groups.

RESULTS: Autophagy levels were significantly different between the groups $(13.7 \pm 5.3,11.7 \pm 3.4$, and $7.5 \pm 3$ $\mathrm{ng} / \mathrm{mL}$, respectively; $\mathrm{p}<0.001)$. In the subgroup analysis, we found significant positive correlations between the level of autophagy and Rentrop score in the Group $1(r=0.463, p<0.001)$.

CONCLUSION: In the present study, autophagy levels were higher in the patients with CAD than in healthy controls. In addition, serum autophagy levels showed a significant positive correlation with the Rentrop score. An increased autophagy level may be considered an important activator and marker of the atherosclerotic inflammatory process in CAD (Tab. 1, Fig. 2, Ref. 20). Text in PDF www.elis.sk. KEY WORDS: autophagy, coronary artery disease, coronary artery occlusion.

\section{Introduction}

Coronary total occlusion (TO) is defined as $\geq 99 \%$ epicardial coronary artery stenosis (1); this process is acute and chronic. Acute TO is seen in myocardial infarction. During and after acute myocardial infarction, several mechanisms play a role in the body.

Occasionally, TO can be seen without an acute coronary syndrome. In these cases, cardiac re-modelling, also known as chronic TO, occurs. The process of cardiac re-modelling involves death or hypertrophy of cardiomyocytes as well as inflammation and fibrosis within the infarct tissue and is associated with disease progression during the chronic stage $(2,3)$.

It is unclear how acute or chronic ischemic events activate and affect autophagy. Previous studies showed that autophagy in the ischemic phase was protective due to an adaptive response of the heart (4-7); these were animal studies and were designed by acute ischemic events. However, a comparison of the autophagy levels in patients with acute and chronic heart disease has not been studied thoroughly.

In the present study, we investigated the role of autophagy in acute and chronic ischemic heart disease as well as whether the role of autophagy exists in the progress of coronary collateral and TO.

${ }^{1}$ Department of Medical Biology and Genetics Istanbul Bilim University, Istanbul, Turkey, and ${ }^{2}$ Department of Cardiology, Istanbul Bilim University School of Medicine, Istanbul, Turkey

Address for correspondence: O. Kaplan, MD, Abidei Hürriyet Cad. No: 166, 34381 Sisli, Istanbul, Turkey.

Phone: +905324609152, Fax: +902123756565

\section{Methods}

\section{Study design}

This study was a prospective observational controlled study.

\section{Study populations}

Between 2016 and 2017, 115 consecutive patients evaluated at the university hospital and diagnosed as having at least one major, chronic, or acute total occlusion of the coronary artery were included in this study. The number of study participants was based on power analysis. All participants were evaluated during coronary angiography (CAG). Participants were divided into three groups: group 1 included patients with chronic TO $(n=49)$; group 2 included patients with acute TO, such as myocardial infarction $(n=36)$; and group 3 included normal controls $(n=30)$. Participants in the groups 1 and 3 had a positive scintigraphy scan or a positive exercise test result. The patients in the group 2 had ST-segment elevated myocardial infarction and underwent a primary percutaneous intervention (PCI). PCI in these patients was performed within 3 hours after the symptoms started, and there were no signs of infection. In addition, patients with uncontrolled hypertension, renal dysfunction, connective tissue diseases, thyroid function disorders, or prior coronary artery disease (CAD) were not included. The study was performed according to the principles of the Declaration of Helsinki, and its protocol was approved by Istanbul Bilim University Hospital Ethics Committee. Informed consent was obtained from all participants.

\section{Biochemical measurements}

After CAG, approximately $2 \mathrm{~mL}$ of blood was drawn from 


\section{3-106}

the median cubital vein from each participant and collected into EDTA-containing tubes. In addition, blood samples of all participants were collected during the coronary angiography process. All samples were centrifuged, and the plasma samples were stored at $-80{ }^{\circ} \mathrm{C}$. The enzyme-linked immunosorbent assay (ELISA) kit for autophagy-related protein 5 (ATG5) in the plasma was studied. ATG5 was evaluated two times for all samples and we used the mean value. Biochemical and haematological parameters were studied on the same day.

\section{Coronary angiography analysis}

All patients underwent CAG by the Judkins technique via a femoral approach. During the procedure, images were recorded at a speed of 15 square/s on a digital angiographic system (ACOM.PC; Siemens AG, Germany). Iopromide (Ultravist 370, Schering AG, Berlin, Germany) was used as the contrast agent. The recordings were examined by two independent cardiologists. Angiographic CAD was defined as $\geq 50 \%$ luminal diameter stenosis of at least one major epicardial coronary artery. CAD severity was determined on the basis of the Gensini score (8). The Rentrop score was used to determine the coronary collateral degree (9). TO was defined as $\geq 99 \%$ epicardial coronary artery stenosis (1).

\section{Statistical analysis}

SPSS for Windows version 15.0 software (SPSS Inc., Chicago, IL, USA) was used for statistical analysis. All continuous variables were expressed as the mean $\pm \mathrm{SD}$, and categorical variables were defined as numbers and percentages. In addition, categorical data were compared using the chi-square test, and continuous variables were compared between the groups using the Student t test or the Mann-Whitney U test, depending on whether they were distributed normally, which was determined with the Shapiro-Wilk test. Furthermore, analysis of variance and least significant difference from post hoc tests were used for intragroup comparisons of the continuous variables. Finally, Pearson and Spearman correlation analysis was used to estimate the relation- ship between the test parameters, and $\mathrm{p}<0.05$ was considered to be statistically significant.

\section{Results}

Overall, 115 participants were enrolled in the present study; 85 had a TO and 30 had a normal coronary artery. Baseline clinical, demographic, and echocardiographic parameters of the participants are listed in Table 1. Age, sex, BMI, smoking status, and dyslipidaemia were similar among the three groups; however, hypertension was different among the groups $(\mathrm{p}=0.036)$.

The results of the left ventricular (LV) echocardiographic and hematologic parameters were significantly different among the three groups (Tab. 1), particularly LV end-diastolic diameter (49.5 $\pm 4.3,50.5 \pm 5.1,47.6 \pm 3.5 \mathrm{~mm}$, respectively; $\mathrm{p}=0.028), \mathrm{LV}$ end-systolic diameter $(34.9 \pm 4,36.6 \pm 5.7$, and $31.7 \pm 3.6 \mathrm{~mm}$, respectively; $\mathrm{p}<0.001)$, ejection fraction $(52.2 \pm 5.2,46.6 \pm 6.7$, and $58 \pm 1.7$, respectively; $\mathrm{p}<0.001)$, white blood cells $(8690 \pm 2061$, $11,943 \pm 2828$, and $7145 \pm 1623$, respectively; $\mathrm{p}<0.001$ ), and haemoglobin $(14 \pm 1.5,14.2 \pm 1.7$, and $12.9 \pm 1.1$, respectively; $\mathrm{p}<0.001$ ).

Autophagy levels were significantly different among the three groups $(13.7 \pm 5.3,11.7 \pm 3.4$, and $7.5 \pm 3 \mathrm{ng} / \mathrm{mL}$, respectively; $\mathrm{p}<0.001$, respectively) (Fig. 1).

The subgroup analysis and comparison revealed that autophagy levels were also significantly different between the groups. Autophagy level was found to be higher when comparing the groups 1 and $2(13.7 \pm 5.3 \mathrm{ng} / \mathrm{mL}$ and $11.7 \pm 3.4 \mathrm{ng} / \mathrm{mL}$, respectively; $\mathrm{p}=$ $0.041)$. The levels of autophagy in the groups 1 and 2 were higher than that in the controls. The autophagy levels of the participants with TO were not significantly different from each other. In contrast, autophagy levels of participants with and without TO were significantly different $(\mathrm{p}<0.001)$ (Fig. 2).

Furthermore, there were no correlations between the level of autophagy and ejection fraction, Gensini score, number of collateral vessels, hematologic parameters. However, significant positive

Tab. 1. Baseline characteristics, laboratory and echocardiographic parameters of the study population.

\begin{tabular}{|c|c|c|c|c|}
\hline Variable & Chronic TO $(n=49)$ & Acute TO $(n=36)$ & Controls $(n=30)$ & $\mathrm{p}$ value \\
\hline Age, years & $62.5 \pm 7.7$ & $58.6 .5 \pm 8.4$ & $60.1 \pm 10$ & 0.105 \\
\hline Gender, female/male & $12 / 37$ & $10 / 26$ & $10 / 20$ & 0.696 \\
\hline $\mathrm{BMI}, \mathrm{kg} / \mathrm{m} 2$ & $25.9 \pm 1.8$ & $26.2 \pm 2.9$ & $27 \pm 1.6$ & 0.107 \\
\hline Dyslipidemia, n (\%) & $22(44)$ & $17(46)$ & $19(63)$ & 0.253 \\
\hline Hypertension, n (\%) & $45(91)$ & $28(77)$ & $29(96)$ & 0.036 \\
\hline Smokers, n (\%) & $37(75)$ & $26(72)$ & $17(56)$ & 0.192 \\
\hline WBC & $8690 \pm 2061$ & $11943 \pm 2828$ & $7145 \pm 1623$ & 0.001 \\
\hline Haemoglobin & $14 \pm 1.5$ & $14.2 \pm 1.7$ & $12.9 \pm 1.1$ & 0.001 \\
\hline Platelet & $246979 \pm 52268$ & $261441 \pm 72731$ & $260200 \pm 51652$ & 0.462 \\
\hline LVEDD, mm & $49.5 \pm 4.3$ & $50.5 \pm 5.1$ & $47.6 \pm 3.5$ & 0.028 \\
\hline LVESD, mm & $34.9 \pm 4$ & $36.6 \pm 5.7$ & $31.7 \pm 3.6$ & 0.001 \\
\hline $\mathrm{LA}, \mathrm{mm}$ & $40.1 \pm 3$ & $40.9 \pm 3.6$ & $39.1 \pm 2.4$ & 0.052 \\
\hline IVS, mm & $11.6 \pm 1.2$ & $11.1 \pm 1.1$ & $11.2 \pm 0.8$ & 0.164 \\
\hline $\mathrm{PW}, \mathrm{mm}$ & $10.8 \pm 0.9$ & $10.4 \pm 0.9$ & $10.4 \pm 0.8$ & 0,115 \\
\hline LVEF, \% & $52.2 \pm 5.2$ & $46.6 \pm 6.7$ & $58 \pm 1.7$ & 0.001 \\
\hline Gensini Score & $67.8 \pm 25.1$ & $68.5 \pm 30.5$ & 0 & 0.053 \\
\hline Autophagy & $13.7 \pm 5.3$ & $11.7 \pm 3.4$ & $7.5 \pm 3$ & 0.001 \\
\hline
\end{tabular}

BMI - body mass index; IVS - interventricular septum; LA - left atrium; LVEDD - left ventricular end-diastolic diameter; LVEF - left ventricular ejection fraction; LVESD - left ventricular end-systolic diameter; PW - posterior wall; WBC - white blood cell count 


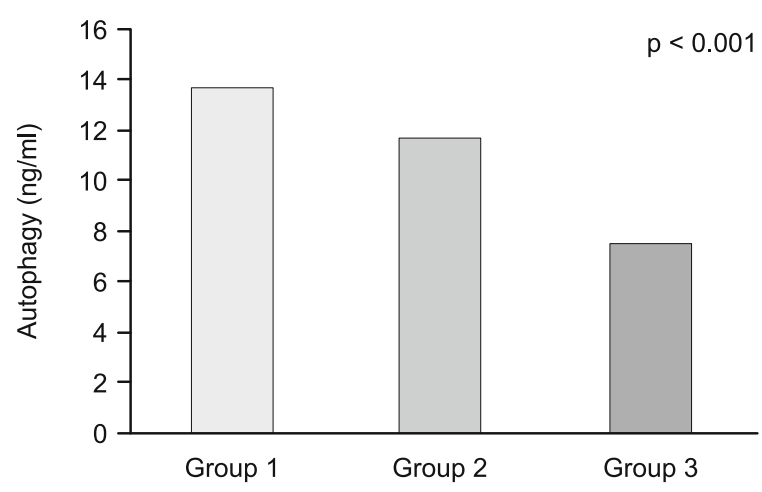

Fig. 1. Autophagy level of groups.

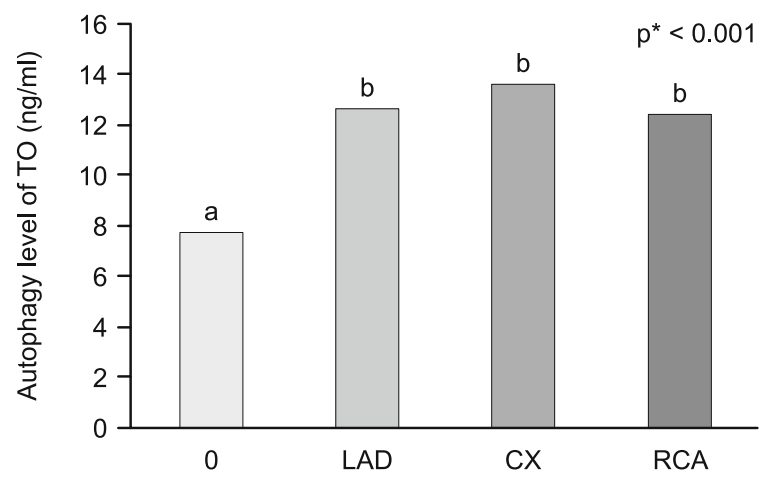

Fig. 2. Comparison of autophagy level of coronary total occlusion. a - normal coronary artery; b - coronary artery with total occlusion, LAD - left anterior descending coronary artery; CX - circumflex coronary artery; RCA - right coronary artery.

correlations were found between level of autophagy and Rentrop score in the group $1(\mathrm{r}=0.463, \mathrm{p}<0.001)$.

\section{Discussion}

The present study showed that autophagy levels were higher in the patients with CAD. Furthermore, patients with chronic TO had the highest autophagy level. In addition, serum autophagy levels showed a significant positive correlation with a degree of collateral. An increased autophagy level may be considered an important activator and marker of the atherosclerotic inflammatory process in CAD.

We are not sure about the aetiology or significance of autophagy during the acute and chronic stages of CAD. Previously conducted animal studies revealed that autophagy protected the heart during the acute ischemic phase (4-7). However, the effect of autophagy on the chronic stages of CAD, such as chronic TO, is unknown. Whether the functional role of autophagy in acute and chronic heart disease (i.e., whether it mediates cell survival or cell death) or it up- or down-regulates cellular function, is yet still poorly understood (10).

Both previous studies as well as our current study showed that autophagy was activated in acute ischemic heart disease, follow- ing ischemia-reperfusion injury and in hibernating myocardium (11-13). If it is seen in hibernating myocardium, autophagy may play a role in chronic TO. Similar results were seen in other studies, with increased autophagy levels in cases of heart failure (10), cardiac hypertrophy (14), and ischemic cardiomyopathy (11). We found that autophagy levels were higher in patients with chronic TO than in those with acute TO. In addition, autophagy occurred constitutively in the normal myocardium. Therefore, we can conclude that autophagy has a significant effect on the chronic heart disease. It works as a protector of the body. Conversely, all patients with chronic TO in our study had coronary collateral. There was a significant positive correlation with degree of coronary collateral, illustrating that autophagy and coronary collateral protect the heart. High autophagy levels may cause a coronary collateral formation. However, it is very difficult to say which autophagy level determines a coronary collateral and protects the body because autophagy is also a mode of cell death that occurs during tissue and organ development to eliminate unnecessary cells (15). Inefficient autophagy or its absence causes the myocardium to perform poorly, and inhibition of starvation-induced autophagy results in cardiac dysfunction and dilatation (16).

We found that LV echocardiographic and hematologic parameters were significantly different among the groups. Both differences of hematologic parameters and structural parameters of the heart can be responsible for different autophagy levels. In addition, several mechanisms might also affect the autophagy levels such as: age, a length of TO, diabetes mellitus and hypertension.

Finally, microbiological studies revealed that autophagy was the basic component of the body and its activation is complex and incompletely understood, leading to the activation of a wide range of signalling pathways (17-20).

\section{Study limitation}

Our study was a single-centre study that included a small study population. Therefore, future studies might include a large patient cohort to overcome these limitations. Furthermore, we were not able to completely evaluate the prognostic value of autophagy levels in the patients with TO.

\section{Conclusion}

We demonstrated that CAD patients had increased autophagy values that were higher in chronic heart disease than in acute heart disease, and autophagy was associated with the degree of coronary collateral. Therefore, evaluating autophagy level in patients with acute and chronic heart disease might provide information regarding the severity of the disease. An increased autophagy level could be considered an important activator and marker of the atherosclerotic inflammatory process in CAD.

\section{References}

1. Shah PB. Management of coronary chronic total occlusion. Circulation 2011; 123: 1780-1784. 
103-106

2. Shan K, Kurrelmeyer K, Seta $\mathbf{Y}$ et al. The role of cytokines in disease progression in heart failure. Curr Opin Cardiol 1997; 12: 218-223

3. Weisman HF, Bush DE, Mannisi JA, Weisfeldt ML, Healy B. Cellular mechanisms of myocardial infarct expansion. Circulation 1988; 78: 186-201.

4. Ma X, Liu H, Foyil SR, Godar RJ, Weinheimer CJ, Hill JA, Diwan A. Impaired autophagosome clearance contributes to cardiomyocyte death in ischemia/ reperfusion injury. Circulation 2012; 125: 3170-3181.

5. Matsui Y, Takagi H, Qu X et al. Distinct roles of autophagy in the heart during ischemia and reperfusion: roles of AMPactivated protein kinase and Beclin 1 in mediating autophagy. Circ Res 2007; 100: 914-922.

6. Kanamori H, Takemura G, Goto $\mathbf{K}$ et al. The role of autophagy emerging in postinfarction cardiac remodelling. Cardiovasc Res 2011; 91: 330-339.

7. Kanamori H, Takemura G, Goto K et al. Autophagy limits acute myocardial infarction induced by permanent coronary artery occlusion. Am J Physiol Heart Circ Physiol 2011; 300: H2261-2271.

8. Gensini GG. A more meaningful scoring system for determining the severity of coronary artery disease. Am J Cardiol 1983; 51: 606.

9. Rentrop KP, Cohen M, Blanke H, Phillips RA. Changes in collateral channel filling immediately after controlled coronary artery occlusion by an angioplasty balloon in human subjects. J Am Coll Cardiol 1985; 5: $587-592$.

10. Takemura G, Miyata S, Kawase Y, Okada H, Maruyama R, Fujiwara $\mathbf{H}$. Autophagic degeneration and death of cardiomyocytes in heart failure. Autophagy 2006; 2: 212-214.

11. Yan L, Vatner DE, Kim SJ et al. Autophagy in chronically ischemic myocardium. Proc Natl Acad Sci USA 2005; 102: 13807-13812.
12. Matsui Y, Takagi H, Qu X et al. Distinct roles of autophagy in the heart during ischemia and reperfusion: roles of AMP-activated protein kinase and Beclin 1 in mediating autophagy. Circ Res 2007; 100: 914-922.

13. Gustafsson AB, Gottlieb RA. Autophagy in ischemic heart disease. Circ Res 2009; 104: 150-115.

14. Hein S, Arnon E, Kostin S et al. Progression from compensated hypertrophy to failure in the pressure-overloaded human heart: structural deterioration and compensatory mechanisms. Circulation 2003; 107: 984-991.

15. Clarke PG. Developmental cell death: morphological diversity and multiple mechanisms. Anat Embryol 1990; 181: 195-213.

16. Kanamori H, Takemura G, Maruyama R et al. Functional significance and morphological characterization of starvation-induced autophagy in the adult heart. Am J Pathol 2009; 174: 1705-1714.

17. Ganley IG, Lam du H, Wang J, Ding X, Chen S, Jiang X. ULK1. ATG13.FIP200 complex mediates mTOR signaling and is essential for autophagy. J Biol Chem 2009; 284: 12297-12305.

18. Kim J, Kundu M, Viollet B, Guan KL. AMPK and mTOR regulate autophagy through direct phosphorylation of Ulk1. Nat Cell Biol 2011; 13: 132-141.

19. Egan DF, Shackelford DB, Mihaylova MM et al. Phosphorylation of ULK1 (hATG1) by AMP-activated protein kinase connects energy sensing to mitophagy. Science 2011; 331: 456-461.

20. Stephan JS, Yeh YY, Ramachandran V, Deminoff SJ, Herman PK. The Tor and PKA signaling pathways independently target the Atg1/Atg13 protein kinase complex to control autophagy. Proc Natl Acad Sci USA 2009; 106: 17049-17054.

Received October 23, 2017. Accepted November 22, 2017. 